\title{
Vers une culture musicale du corps
}

\section{Lothaire Mabru}

\section{(2) OpenEdition \\ Journals}

Édition électronique

URL : http://journals.openedition.org/ethnomusicologie/106

ISSN : 2235-7688

Éditeur

ADEM - Ateliers d'ethnomusicologie

Édition imprimée

Date de publication : 1 décembre 2001

Pagination : 95-110

ISBN : 2-8257-07-61-9

ISSN : 1662-372X

Référence électronique

Lothaire Mabru, "Vers une culture musicale du corps », Cahiers d'ethnomusicologie [En ligne],

14 | 2001, mis en ligne le 10 janvier 2012, consulté le 30 avril 2019. URL : http://

journals.openedition.org/ethnomusicologie/106

Ce document a été généré automatiquement le 30 avril 2019.

Tous droits réservés 


\title{
Vers une culture musicale du corps
}

\author{
Lothaire Mabru
}

1 La question du geste, et plus globalement celle du corps dans la musique commence depuis quelques années à susciter des travaux de recherche, mais il faut bien avouer qu'elle est encore aujourd'hui un terrain sinon neuf, du moins peu exploré. Pourtant, dès 1936 André Schaeffner, dans son ouvrage désormais classique, intitulé Origine des instruments de musique. Introduction ethnologique à l'histoire de la musique instrumentale avait largement ouvert la voie, en postulant pour une origine corporelle de la musique, contre la thèse de l'origine linguistique. Ce faisant, il montrait l'importance du corps dans la pratique musicale et avançait des notions d'une grande pertinence, telle que celle de «lisibilité corporelle» de la musique, dont la portée n'a pas été assez mesurée, me semble-t-il. Mais André Schaeffner opposait les « musiques primitives » à notre « musique des 'conservatoires', musique trop souvent de papier» (1936: 11), reconduisant ainsi l'inévitable opposition oralité/écriture. D'où la question d'un dosage différent du poids du corps dans les diverses modalités de la transmission et de la fixation du savoir. Jean Molino, dans un article relativement récent (1988), opposait lui aussi les pratiques musicales sans écriture à celles qui l'utilisent, en plaidant encore une fois pour une moindre importance du corps dans le cas des secondes. J'ai montré ailleurs (Mabru 1999) qu'il n'en était rien, à travers l'exemple du violon dans la musique dite savante. Quoi qu'il en soit, l'article de Molino permettait de renouer avec cette question et il mettait en évidence la nécessité de redonner au corps la place qu'il a toujours eue mais que, du fait de la construction dans notre culture de la notion de musique comme pureté sonore, on avait la fâcheuse tendance à occulter. Peu de travaux ont été suscités par cette contribution capitale de Molino. Et lorsque la question du corps est traitée, c'est le plus souvent dans une perspective psychologisante, avec notamment la notion de geste intérieur qui a fait fortune, et ne me paraît pourtant pas d'une grande utilité. La musique n'est pas une entité pure (comme le fait si justement remarquer Jean Molino) qui aurait quelque emprise sur le corps, mais je dirai plutôt qu'elle est un comportement: un comportement soumis à certaines règles et contraintes, déterminant ainsi ce que j'appelle une culture musicale du corps. En effet, nul ne doute du caractère culturel du comportement du musicien. Pour s'en convaincre, il suffit de penser à la tenue du violon 
dans différentes pratiques musicales : le violoniste indien joue assis en tailleur, le violon tenu entre la poitrine et l'un des pieds, le violoniste dit classique se tient debout, avec le violon sur la clavicule et maintenu horizontalement. Le ménétrier de village des siècles passés le porte à hauteur de poitrine etc. Et je ne considère ici que la tenue de l'instrument, au sens strict de prise en main, mais il faut aussi prendre en compte toute la gestuelle du musicien - qu'il soit instrumentiste ou chanteur - qui concerne aussi bien les mouvements nécessaires à l'obtention des sons que la dynamique du corps dans son entier.

$2 \mathrm{Si}$, dans les pratiques actuelles de la musique, ce que l'on nommera (en réactivant la notion jadis forgée par Marcel Mauss (1985) de techniques du corps) les techniques du corps musicien semblent naturelles et paraissent n'obéir qu'à des impulsions intérieures, il n'en demeure pas moins qu'elles relèvent d'une incorporation (terme préférable me semble-t-il à celui d'intériorisation) de règles comportementales culturellement déterminées et s'inscrivant dans une histoire du corps. Aussi, dans les pages qui suivent, je souhaite montrer comment s'est constituée dans notre société cette culture musicale du corps.

\section{Une histoire longue}

3 L'objet que l'on entend questionner ici, la conjonction du corps et de la musique, impose de lui-même les limites chronologiques de l'investigation. En effet, c'est aux XVIe et XVIIe siècles que s'intensifie et se développe la pratique musicale instrumentale dans la société européenne ; et c'est à cette même époque que la question du corps dans l'espace public reçoit une attention particulière. Si, comme l'a fait remarquer Norbert Elias, la question des convenances corporelles a toujours fait l'objet de débats, au XVIe siècle elle se voit renouvelée, avec notamment la parution du traité de Didier Erasme de Rotterdam, qui "marque un changement et constitue la concrétisation de certains processus sociaux » (Elias 1973: 79). Autrement dit, se constitue un nouvel espace du corps, qui affectera progressivement toutes les couches de la société. Je poserai donc en préalable la question du corps dans l'espace public de façon globale, dans la société de l'Ancien Régime, pour situer historiquement le problème. En effet, toujours selon Elias, le nouvel espace du corps qu'instaure le traité d'Erasme se développera tout le long de l'Ancien Régime. Autrement dit, une culture du corps se constitue qui conduira à une normalisation des comportements sur une longue durée. Et si les couches supérieures de la société sont d'abord les seules concernées, le processus touchera progressivement l'ensemble de la population, avec bien sûr des variations.

4 Mais la civilité ne sera pas le seul lieu de prise en charge du corps. Le XVIIe siècle est aussi l'âge de l'éloquence qui englobe aussi bien « l'art de la harangue, l'art de la conversation, sans compter la "tacita significatio» de l'art du geste " (Fumaroli 1994: 30). Aussi, et dès lors que la musique vocale et le langage auraient une origine commune, comme le veut la vulgate lettrée, il faudra considérer la question de la rhétorique. Et si l'on peut parler d'une culture du corps éloquent, celle-ci ne serait-elle pas passée dans le domaine musical? Pour examiner la question il convient de considérer les différents lieux d'exercice de la voix : le barreau et la chaire, mais aussi le théâtre, qui distingue diverses modalités vocales : la déclamation, le récitatif, le chanter. Du parler au chanter, pourra-telle passer au jouer? 
Fig. 1 : « Maître à danser » portant son violon sur le bras.

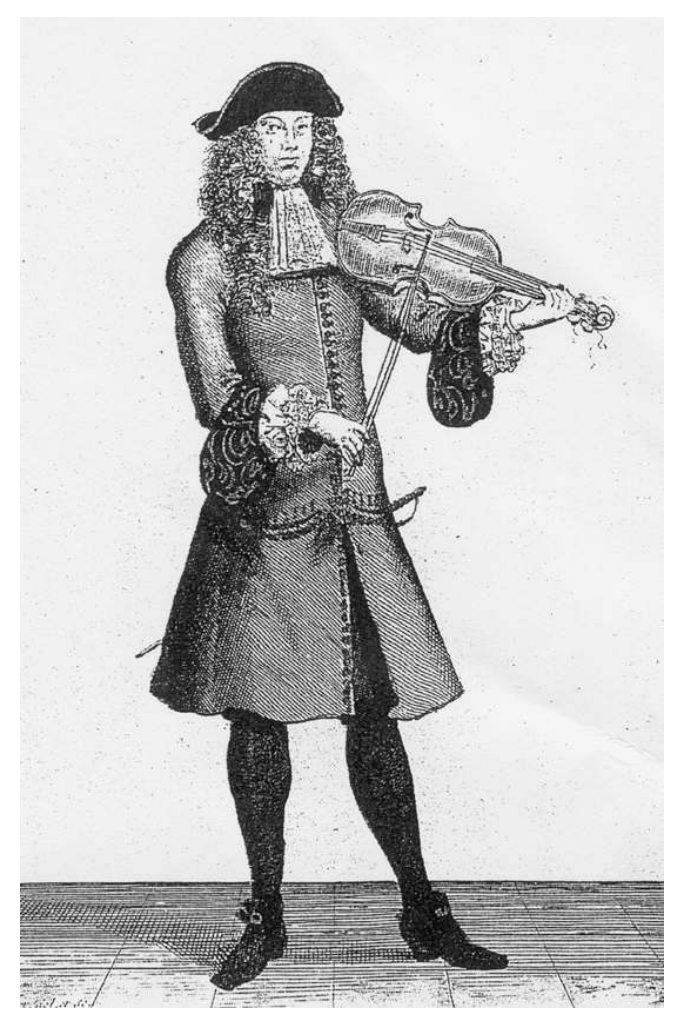

Gravure de N. Bonnart (1682)

\section{Convenances et contenances}

5 «Externum corporis decorum»: la civilité corporelle a pour objet les convenances extérieures du corps. La période qui s'étend du XVIe au XVIIIe siècle peut se comprendre, nous dit Elias, comme une phase de transformation des comportements, cela du fait de la reconfiguration sociale:«Ce sont les bouleversements sociaux, la refonte des relations humaines qui finissent par entraîner des changements: on ressent plus que naguère l'obligation de s'imposer l'autocontrôle "(1973: 117). Cette nécessité d'un autocontrôle révèle l'importance que l'on accorde au regard de l'autre, désormais déterminant dans le comportement de l'homme. La multiplicité des rencontres, le brassage des classes sociales, engendrent de nouvelles normes de conduites, en un mot, des convenances, ici corporelles. Une économie du corps, exposé au regard de l'autre, se met en place par un dressage de ce corps: d'un dressage du corps qui s'opère en toute conscience, pour ensuite laisser la place à des comportements qui seront qualifiés d' « intériorisés ", et que l'homme conçoit dès lors comme naturels. Et l'on comprend alors que les manuels de civilité peuvent sembler aujourd'hui naïfs, voire surprenants, dans la description de conduites devenues naturelles. En d'autres termes ces manuels et traités ont élaboré des techniques du corps, selon le sens qu'en donne Marcel Mauss.

6 Mais avant de devenir techniques du corps, c'est à dire comportements reçus comme naturels, il a fallu un long travail de dressage, travail que décrivent les manuels et traités de civilités. En effet, aux XVIIe et XVIIIe siècles, ces ouvrages détaillent précisément le comportement que doit adopter l'honnête homme. Mais «il n'est pas question de la 
bonne grâce ou d'un certain air et attrait qui est naturel dans les actions de certaines personnes, lesquelles ont un talent particulier de la nature pour plaire en tout ce qu'elles font [...]. On ne saurait donner de préceptes pour acquérir cet heureux agrément, puisque c'est une libéralité de la nature. Mais comme c'est fort peu de choses de plaire seulement aux yeux du corps, si nous n'avons en même temps le bonheur de plaire aux yeux de l'âme, ce n'est pas aussi ce charme extérieur que nous devons rechercher, comme le véritable principe de la politesse : nous devons aspirer à quelque chose de plus solide qui marque la bonne disposition du dedans, plutôt que la bonne disposition du dehors »(Courtin 1699 : 1-2).

7 L'essentiel du projet de la civilité est dit : s'il faut travailler son corps, ses comportements, c'est parce que l'enveloppe charnelle de l'homme, son extériorité, n'est pas seule en cause et renvoie à son intériorité. Le corps, ce qui est donné à voir donc, n'est rien en lui-même, s'il ne renvoie pas à la part spirituelle de l'homme. Si cette dernière ne peut être directement atteinte, au moins le corps qui la contient sera la seule voie d'accès possible. Toute la civilité postule que ce qui est donné à voir en extérieur, renverra automatiquement à l'intérieur de l'homme : le visible témoignerait de l'invisible. Il y a des convenances, c'est-à-dire des choses qui se font et d'autres qui sont à exclure, parce que le corps et sa dynamique renseignent sur l'âme humaine. Aussi, pour être jugé favorablement par autrui, il faut savoir se contenir : «La contenance n'est autre chose que l'accord du dedans avec le dehors d'un homme, c'est à dire de la personne avec la chose, le lieu et le tems (sic) dont il s'agit [...]. Le mot même de contenance l'exprime tout seul, en ce que venant du mot contenir, une personne n'est censée avoir de la contenance que parce qu'elle contient en premier lieu ses passions et puis ses membres ou ses actions, sa langue ou ses paroles dans les bornes où toutes choses doivent être pour répondre à ces circonstances "(ibid. : 237-238).

8 Convenance et contenance sont ainsi étroitement liées: se contenir c'est se mettre en adéquation avec les circonstances dans lesquelles on se trouve. Et de Courtin, rappelant la civilité des Anciens indique les quatre règles à observer : « $1:$ se conduire selon son âge et sa condition, 2 : prendre garde à la qualité de la personne avec laquelle on se trouve, 3 : bien observer le temps, $4:$ regarder le lieu où on se rencontre » (ibid. : ch. 2, p. 3).

9 Les règles comportementales sont étroitement dépendantes de l'inscription corporelle dans une dimension spatio-temporelle : l'ici et le maintenant déterminent la conduite. Les manuels de civilité le répéteront à l'envi tout le long de l'Ancien Régime, et même audelà : «Il est cependant à propos de composer son visage selon les circonstances où l'on se trouve et les personnes avec lesquelles on converse ». Peut-on lire dans l'édition de 1845 de la civilité chrétienne de J.-B. de la Salle (1945: 384). Autrement dit il faut savoir être "maître de ses humeurs et de ses mouvements» (Callières 1717:4) en un mot « contenir ses passions » (Courtin 1699). Pour y parvenir, le seul moyen sera la recherche de la mesure en toutes choses, du juste milieu, catégorie morale empruntée aux Anciens, et plus particulièrement à Cicéron, souvent cité dans les traités de civilité. La définition de la notion de decorum donnée dans le De officiis - «la tempérance et la modération, toute maîtrise des troubles de l'âme et la mesure en toutes choses " (1974: 152) - ne résume -t-elle pas à elle seule le projet de la civilité ? Ne pourrait-elle avoir un quelconque rapport avec la pratique musicale, surtout lorsque l'on sait que cette catégorie morale a directement à voir avec l'esthétique ? Car pour Cicéron le décorum est une catégorie morale, mais de la beauté morale. 
10 Les traités de civilité de l'Ancien Régime prennent peu en compte la question de la pratique musicale. Mais les quelques commentaires que l'on peut glaner à ce propos méritent que l'on s'y arrête. Ils permettent de montrer que l'espace des convenances qui s'élabore à cette époque ne concerne pas les seules relations sociales, mais transcende tous les domaines de la vie en société. Ainsi, Antoine de Courtin consacre un chapitre à la question de savoir " s'il faut chanter ou jouer des instruments" $(1699, \mathrm{ch}$. XV : 1). Selon lui le seul fait de pratiquer un instrument peut être un écart à l'impératif de modération, puisqu'il met en valeur celui qui possède ce talent. Mais dès lors que le musicien se trouve dans l'obligation de jouer, il doit le faire avec une certaine réserve corporelle: «Et surtout, il ne faut ni tousser trop ni cracher, ni être trop longtemps à accorder sa guitare ou son luth. Il faut bien se garder aussi, de se louer soi-même par certains gestes étudiez (sic), qui marquent votre complaisance, et dire par exemple, lorsque l'on chante, voilà un bel endroit, en voici encore un plus beau, prenez garde à cette chute, etc... cela est de l'homme vain, ou de peu. Il faut aussi avoir soin de finir promptement, pour éviter d'être ennuyeux, et pour laisser, comme on dit, la compagnie sur sa bouche. Et même il faut finir d'autant plus tôt, que personne ne vous dira, c'est assez; parce que c'est une incivilité de le dire, si celui qui chante est personne de condition ; comme ç'en est une de parler et de l'interrompre quand il chante » (1699: 1-2). Jean-Baptiste de la Salle ira plus loin dans les recommandations concernant les gestes : "Il n'est pas non plus bienséant de faire de certains gestes qui marquent la cadence; c'est ce qu'il n'est pas aussi bien de faire lorsqu'on joue de quelqu'instrument » (1736: 128). Dès lors que l'honnête homme pratique un instrument de musique, son comportement doit être réservé. Ce qui permet d'expliciter certaines des tenues du violon : celle du ménétrier - musicien de la danse - de l'extériorité donc, et celle de l'honnête homme qui doit intérioriser sa conduite par une réserve corporelle. Au salon la retenue et la réserve, au bal et dans la rue, la gesticulation et l'indécence? L'iconographie semble plaider pour une telle distinction. A.P. de Mirimonde oppose ainsi deux types de tableaux, ceux qui donnent à voir « des violoneux plus ou moins dépenaillés », et ceux qui montrent « un élégant violoniste » (1977: 50-51).

11 Quoi qu'il en soit, la musique doit être arrachée du corps et déléguée dans les seules actions nécessaires à la manipulation de l'instrument. Le musicien ne saurait se laisser entraîner par sa passion de jouer ; il lui faut rester « naturel », c'est-à-dire ne pas montrer la nécessité de l'effort que demande la pratique musicale : «Il est aussi de très mauvaise grâce d'avoir des manières de chanter qui soient ou grossières, ou affectées et singulières. Le moyen de bien chanter, agréablement, c'est de le faire d'une manière qui soit tout à fait naturelle " (de la Salle 1736: 129). Le naturel dans le comportement rejoint ici la double notion de convenance/contenance, dans une recherche d'un juste milieu entre laisser-aller et affectation. Laisser-aller: ne pas faire attention à ce que l'on fait. Affectation : faire trop attention à ce que l'on fait. Le naturel de la civilité repose sur un paradoxe, puisqu'il faut se contrôler pour éviter le laisser-aller, mais ne pas se contrôler pour éviter l'affectation. Toute la difficulté du comportement en société réside dans cette quête constante de l'équilibre, ou comme dirait Ervin Goffman : « la vie, c'est sur le fil » (1974 : 121).

12 De plus, les prescriptions comportementales des ouvrages de civilité ne concernent pas les musiciens de métier, mais plutôt les « honnêtes gens » qui pratiquent un instrument pour leur plaisir. La «modestie que l'on doit faire paraître dans le maintien du corps » selon la formule de J.-B. de la Salle, ne concerne pas le professionnel : « Les grands gestes, dans le chant, ne conviennent qu'à des acteurs; dans un cercle privé, on doit conformer 
doucement les gestes aux paroles et aux gradations de la voix ; car il serait ridicule d'être, en chantant, aussi immobile qu'une statue. Ce que l'on dit ici de la voix regarde aussi les instruments » (de la Salle 1845 : 406).

13 Entre gesticulation et immobilité se situe la modestie, comme culture du juste milieu. De plus, il y a des techniques du corps différentes selon le statut du musicien, mais aussi selon le lieu d'exercice de ses talents, comme cela semble être ici le cas avec le violon ${ }^{1}$. Pour lors, retenons la réserve corporelle imposée au corps de l'honnête homme musicien, et considérons maintenant le rapport du corps et de la parole

\section{L'éloquence du corps}

L'on retrouve ici Erasme de Rotterdam qui, avec l'Ecclesiastes de 1535 marque le renouveau de l'intérêt pour l'actio rhétorique, en réactivant Quintilien et Ciceron (Fumaroli 1994 :106-110). Dès lors une place importante sera redonnée à cette partie de la rhétorique qui prend en charge la dimension corporelle de l'orateur. Si le langage du corps est un lieu commun, pour les rhéteurs ce langage se travaille. Car il s'agit ici de convaincre son auditoire, de le persuader et de l'émouvoir, et pour cela le corps et sa dynamique ne sauraient être négligés: «Le geste n'est pas de petite importance à un homme qui parle en public pour faire passer les pensées et les passions de son esprit en celui des auditeurs » (Conrart $1657: 187)$.

On comprend dès lors la profusion de traités de rhétorique, d'art du bien parler, aux XVIIe et XVIIIe siècles, et même au-delà. La lecture de certains d'entre eux, parmi les plus connus, permet en premier lieu de constater des points communs avec la civilité. Ainsi, dans cette définition de l'action par Jean Beauvais : «L'action est une obéissance de tout le corps qui se conforme au sujet que l'on va répéter, et prend toutes les attitudes que le discours exige pour exprimer les différentes passions qu'on y dépeint. Il faut observer que l'action ne doit avoir en elle-même rien d'efféminé, d'incivil, de rude, ni de trop brusque. Enfin, elle ne doit être ni trop hardie, ni trop timide » (Beauvais 1784 : 473). Dans les deux cas l'attention est focalisée sur l'apparence, sur ce qui est donné à voir dans l'espace public; la recherche de l'équilibre, du juste milieu est un but commun. Et si pour la civilité l'extérieur donne à lire l'intériorité de l'homme, pour la rhétorique, la dynamique du corps est liée au contenu du discours : " Les sentimens, les affections de l'âme ont un ton de voix, un geste et une mine qui leur sont propres. Ce rapport des choses et de la manière de les prononcer fait les bons déclamateurs » (Lamy $1715: 429)$. Ou bien encore : "Le geste suit naturellement la voix, et se conforme comme elle, aux sentiments de l'âme. C'est un langage muet, mais éloquent, et qui souvent a plus de force que la parole même » (Rollin 1755 : 616).

16 La rhétorique distingue deux types de parole vive: la parole ordinaire, et la parole éloquente, celle des professionnels du discours, si l'on peut s'exprimer ainsi. Si la première relève de la "nature ", la seconde sera un art qui devra respecter cette nature. Pour ce faire, la rhétorique va élaborer une véritable sémiologie des gestes - ceux-ci étant les signes naturels des passions - et développer des techniques précises et détaillées du corps éloquent, qui visent aussi bien le corps en son entier que chacune de ses parties. Ainsi, par exemple, J. Beauvais passera en revue les « défauts et propriétés » de la tête, du front, de l'œil, de la bouche, des pieds, des mains et des bras, pour ensuite considérer la « conduite du corps en son entier». 
17 Dans cette conduite du corps en mouvement, la rhétorique, qui reconduit les recommandations de la civilité en prônant la quête du juste milieu, ajoute au comportement civil une dimension expressive. Mais si le geste exprime un sentiment, il doit surtout convaincre l'auditoire de la réalité et de la sincérité de ce sentiment. Le bon orateur sera celui qui maîtrise bien les passions et connaît les gestes convenant à chaque sentiment, mais aussi celui qui pourra se comporter naturellement: "Tout doit être étudié dans un homme qui parle en public, son geste, son visage; et ce qui rend cette étude si difficile, c'est que si elle paraissait, elle ne ferait plus son effet. Il faut employer l'art et il n'y a que la nature qui doive paraître ; aussi c'est elle qu'il faut étudier [...]. Jamais orateur ne réussit que quand il a acquis d'être ainsi naturel. Il peut dire ce qu'il a appris par mémoire, mais il faut qu'il paraisse le faire, comme si la nature seule sans art et sans préparation le faisait parler » (Lamy $1715: 429$ ).

18 Tout le talent de l'orateur, dans le domaine de la conduite de son corps, consiste à une maîtrise telle qu'il semble ne pas songer à ce travail des gestes. Au naturel paradoxal de la civilité correspond le naturel étudié de la rhétorique. Si l'homme civilisé recherche l'équilibre entre laisser-aller et affectation, jusqu'à l'effacement de son corps, l'orateur doit s'en servir comme d'un outil de persuasion et de communication des émotions, tout en gommant son statut d'outil. Dans les deux cas un travail sur le corps s'accomplit, mais avec des finalités différentes. Car l'effacement du statut d'outil du corps éloquent n'est pas un effacement de ce corps, mais une mise en scène de sa dynamique dans l'espace public.

19 Que l'orateur recherche le « naturel » dans l'action, ne saurait le faire taxer d'insincérité. D’abord, et selon le précepte de Cicéron souvent cité dans les ouvrages de rhétorique, l'orateur doit être convaincu lui-même de ce qu'il dit s'il veut convaincre son auditoire. Ensuite parce que l'orateur n'est pas l'acteur de théâtre, lequel « ne vit pas, il imite un comportement et des passions; quand même ce comportement serait vertueux et ces passions nobles, la fiction les régit et les fausse » (de Rougemont, 1981 : 330).

Autrement dit le théâtre serait le lieu de l'exagération dans l'action, tandis que la chaire ou le barreau seraient ceux du naturel, donc de la vérité. Il y aurait en somme une culture $\mathrm{du}$ corps éloquent différente de celle du corps comédien. Les traités de rhétorique composés au XVIIe siècle le répètent à l'envi: l'orateur ne saurait calquer son comportement sur celui, outré, de l'acteur de théâtre. Il y aurait bien ainsi des techniques du corps différentes en fonction des lieux et des finalités, ici convaincre ou divertir. Y aurait-il des techniques du corps différentes selon les modalités vocales: parler, déclamer, chanter? 
Fig. 2 : La tenue du violon

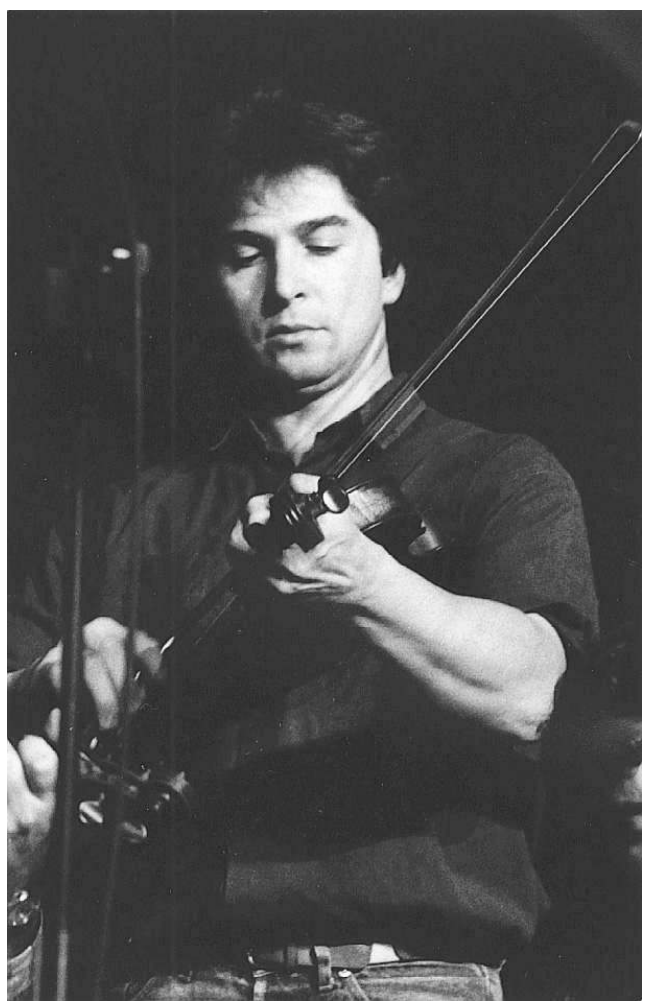

a) La tenue «ménétrière ». S. Barran, membre de l'Ensemble des Violons de Gascogne. Bal à Colomiers, 1993

(photo : Lothaire Mabru)

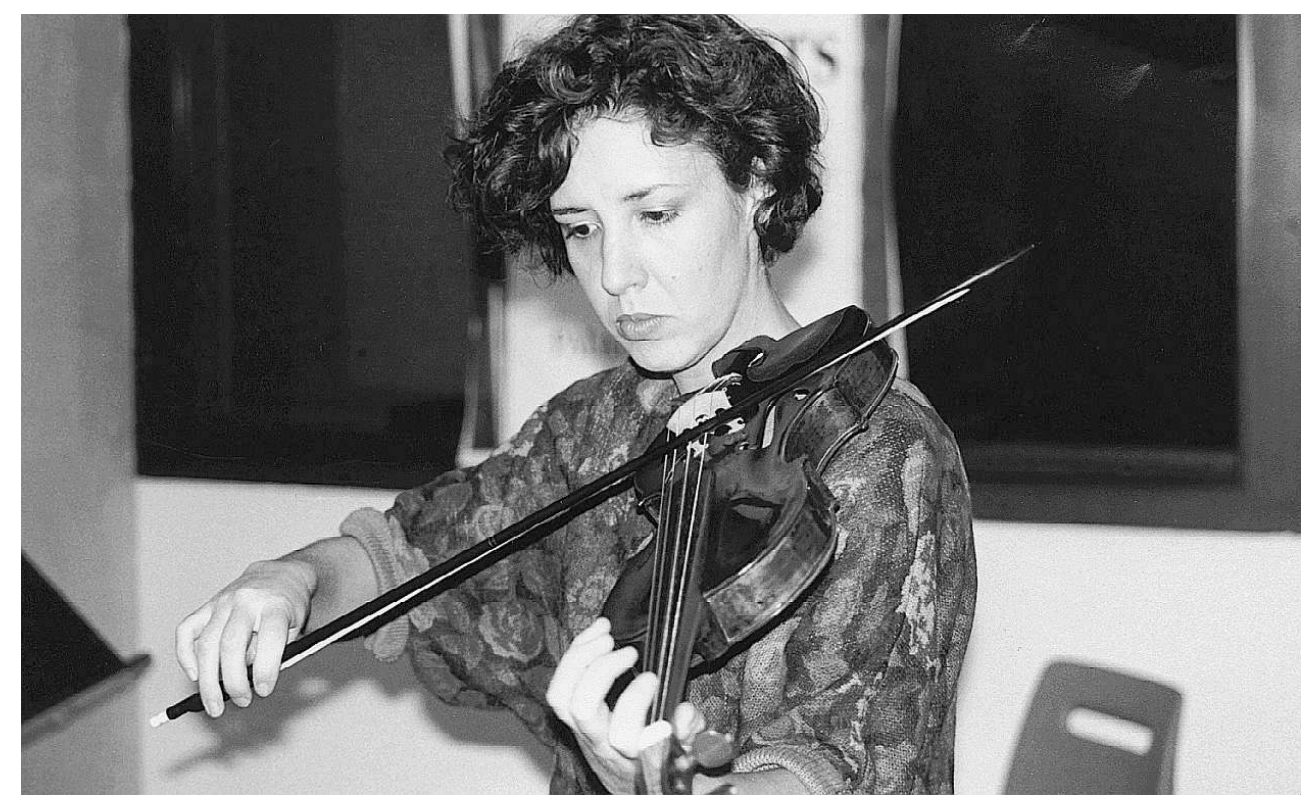

b) La tenue baroque. Isabelle Duluc, Bordeaux, 1994

(photo : Lothaire Mabru) 


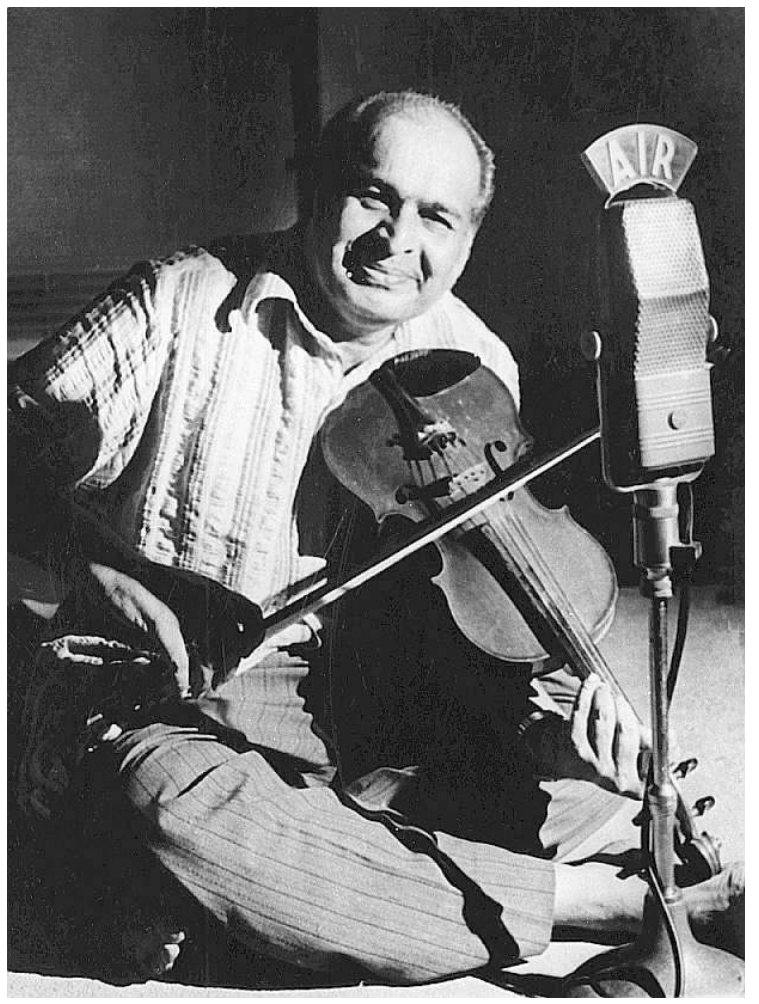

c) La tenue indienne : V. G. Jog à All India Radio, s.d. (photo : Christian Ledoux).

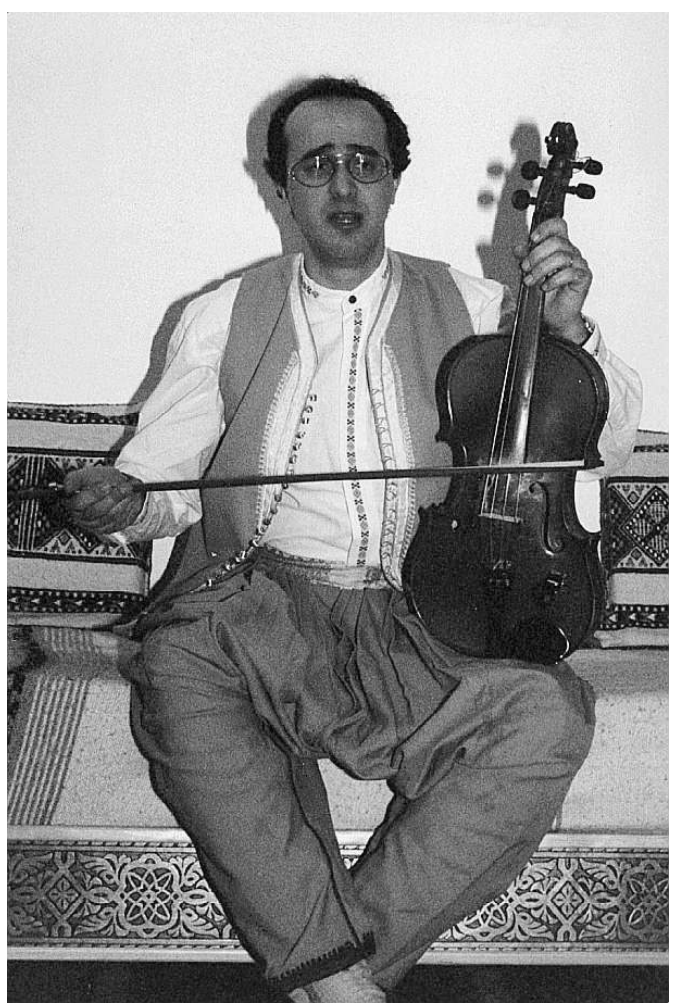

d) La tenue maghrébine : Taoufiq Bestanji, Constantine, Algérie (photo: x.). 


\section{Du parler au chanter : lieux et techniques du corps}

«Plusieurs s'imaginent que le chant tenant de la déclamation, et ayant pour but d'exprimer les passions, doit être exécuté avec beaucoup d'affectation, que d'autres appelleraient outrer le chant. Pour moi je tiens que ce n'est pas avoir ajouté au chant que cette grande affectation qui souvent est accompagnée de grimaces, si ce n'est pour le récitatif, je veux dire pour le théâtre. Mais pour le chant qui se pratique dans les ruelles, je soutiens que c'est ajouter de l'agrément que d'en retrancher cette façon de chanter trop ampoulée, qui en ôte toute la mignardise et toute la délicatesse » (Bacilly 1679 : 11). Cet extrait de L'art de bien chanter le dit clairement : le comportement du chanteur dans les ruelles ${ }^{2}$ ne saurait prendre pour modèle celui des acteurs. Seules les règles de la civilité semblent convenir au chanteur de salon, qui doit faire montre d'une réserve corporelle. En tous cas un fait est certain : le lieu de profération de la parole détermine bien le comportement du locuteur, qu'il parle ou qu'il chante

Pourtant les choses vont évoluer au début du XVIIIe siècle, et les préceptes de la rhétorique vont passer dans les pratiques artistiques, théâtrales et musicales. Une réconciliation qui va affecter le chanteur a lieu entre l'orateur et le comédien. On la doit à Grimarest qui, dans son Traité du récitatif (1707), formule une théorie de la déclamation théâtrale. Pour ce faire, il s'appuie explicitement sur la rhétorique, et notamment sur celle de R. Bary et de Le Faucheur, comme l'ont montré Peter France et Margaret Mc Gowan (1981: 303-317) ${ }^{3}$. Ce traité ne resta pas lettre morte, mais occupa une place de choix dans les bibliothèques des comédiens du XVIIIe siècle. Mais surtout, Grimarest met en relation différentes économies corporelles selon le lieu et la finalité de la parole. Ainsi, de la lecture que l'on fait dans les salons : «Quand on fait une lecture, ce n'est point pour se donner en spectacle: ainsi on ne doit pas allier le geste avec la prononciation. Néanmoins les différentes passions que l'on exprime, exigent naturellement de petits mouvements de bras et de visage qui donnent du feu et de l'agrément : on ne peut les refuser à la nature qui nous les fait faire involontairement » (1707: 100).

La réserve corporelle est toujours de rigueur, mais le corps peut accéder au naturel de la rhétorique. Ce n'est qu'au théâtre que l'acteur et le chanteur pourront développer une gestuelle plus démonstrative, cela du fait des dimensions spatiales. L'acteur comme le chanteur ne gesticuleront certes plus, mais se conformeront à la gestuelle de l'orateur. Pour Grimarest la déclamation, régie par les lois de l'actio, est une partie de la rhétorique qui concerne la voix et le geste, et s'adresse aussi bien à l'orateur qu'au comédien, qu'il parle ou chante (1707: 119-122). Et l'on comprend ainsi que Jean Blanchet, quelques décennies plus tard, puisse proposer dans ses Principes philosophiques $d u$ chant destinés aux maîtres de chant la notion d'action chantante, jusqu'à lui consacrer un chapitre entier ${ }^{4}$. Nul doute que la rhétorique est passée dans le domaine musical, pour lors vocal : «L'action en général, telle qu'on doit l'entendre ici, est l'art de peindre le sentiment par le mouvement de certains membres, par l'air du visage et par tout le maintien du corps. On peut regarder les gestes comme une langue naturelle et universelle. [...]. L'action chantante est l'art dont il s'agit, appliqué aux paroles mises en musique : aussi le jeu des acteurs doit varier autant que ces dernières, ou plutôt autant que les passions exprimées par les paroles, et elle doit emprunter leur caractère. C'est pourquoi l'on doit faire briller sur nos théâtres lyriques, tantôt des grâces fières, tantôt des grâces ingénues, tantôt des grâces 
sérieuses, tantôt des grâces enjouées; quelques fois des grâces vives et piquantes, et d'autres fois des grâces négligées et tendres » (Blanchet 1766 : 78-78).

A la suite de quoi Blanchet développera cette notion d'«action chantante», par l'établissement d'un catalogue des techniques du corps chantant. A l'instar de Grimarest, il prendra soin au préalable de distinguer des degrés dans la dynamique du corps, selon le lieu du chant: «On comprend que le jeu d'un chanteur doit être exagéré au théâtre, et qu'il doit être moins chargé que celui d'un simple déclamateur; la raison en est que l'on insiste davantage sur les sentiments dans le chant que dans la déclamation de la tragédie ou de la comédie. On conçoit aussi que ce jeu doit être beaucoup moins animé et chargé hors de nos théâtres : presque toute l'action de celui qui chante dans les compagnies doit être sur son visage » $(1766: 78)$.

La réserve corporelle est toujours indiquée dans les cercles intimes car le chant porte en lui les sentiments, mais le chanteur peut et doit investir son corps comme élément de la performance musicale: selon Blanchet l'action chantante "peut se réduire aux mouvements des bras et des yeux et à l'air du visage " (ibid.). A la suite de quoi, il va établir un catalogue des différents mouvements, en fonction des passions qui doivent leur correspondre, tel un véritable traité d'actio musicale. Mais il y a plus : les gestes ne sont pas les seuls concernés par ce domaine qui englobe aussi les «contenances»: «Outre les mouvements dont j'ai parlé, il est de certaines situations de tout le corps, ou plutôt de contenances terribles ou gracieuses, fières ou naïves etc... on les appelle attitudes. Un chanteur doit encore plus s'en servir que les gestes; les premiers font un tableau entier, les seconds ne sont qu'un coup de pinceau. On voit que l'action peut être considérée comme une certaine éloquence du corps, et qu'elle est une espèce de langage dont l'âme se sert pour exprimer ses passions » $(1766: 83)$.

26 Avec la notion d'action chantante, Jean Blanchet propose une articulation entre un contenu - celui du chant - avec une forme, ici corporelle. D'une articulation qui, comme en rhétorique, relie l'intérieur (l'âme et ses passions) avec un extérieur, le corps. Ce n'est plus ici seulement une question de contenance et de convenance, mais de contenance et de contenu. Et d'une articulation qui doit reposer sur l'observation des hommes. Quels sont les meilleurs observateurs de l'homme sinon les peintres et les sculpteurs? On comprend dès lors que, pour Blanchet, les plus célèbres chanteurs sont ceux qui « ont savamment étudié les chefs d'œuvres de peinture et de sculpture: ils doivent à des observations fines et profondes en ce genre la belle entente de leurs vêtements, la noblesse de leur geste, les nuances de leurs expressions, les grâces et la variété de leur jeu » (ibid.).

27 On le voit, l'action chantante ne saurait se résumer à quelques mouvements des bras et de la tête, mais concerne la globalité du corps chantant, comme tableau des sentiments de l'âme. Blanchet lui-même n'avait-il pas écrit précédemment que le chant «est une peinture animée, parlante et chantante, et qu'il est très capable de représenter toute la durée d'une passion quelconque" $(1764: 342)$ ? Nulle coupure n'est possible ici entre l'aspect auditif et visuel de la musique, dès lors qu'il s'agit de convaincre le spectateur de la passion peinte par la musique. C'est à dire de le toucher.

En ouvrant la porte du chant à l'actio rhetorica, Blanchet ne fait que réaliser un souhait de son époque qui, relisant les Anciens, regrettait que soit tombé en désuétude cet art du geste $^{5}$. Mais s'il y a bien dès lors ce que j'appellerai un comportement musicien du chanteur, qu'en est-il dans la pratique musicale instrumentale? Car si Blanchet a pu forger la notion d'action chantante, c'est bien en vertu de l'idée communément partagée 
au XVIIIe siècle, que musique vocale et langage sont dès l'origine de l'humanité intrinsèquement liés. S'il y a une action du corps parlant, il doit y avoir une action du corps chantant. De plus, si la musique instrumentale est appréciée, elle n'est guère estimée, comme le montre Belinda Cannone (1990: 26) et le chant, imitation de la nature, demeure le paradigme de toute musique. Pour lors, estimons le chemin parcouru: une culture du corps se met en place aux XVIIe et XVIIIe siècles avec le développement de la civilité et la réactivation de l'actio. Toutes deux concourent à forger un comportement du corps chantant, qui peut varier en fonction des lieux de la performance musicale. Mais le corps est toujours partie prenante dans la réalisation de la musique.

\section{Vers une actio musicale instrumentale}

Peut-on envisager le développement d'une actio musicale généralisée, sachant que la musique instrumentale est sous l'emprise de la musique vocale, qu'on la considère comme son imitation ${ }^{6}$ ? David Barnett, étudiant il y a quelques années le passage de la rhétorique dans la musique, montre que ses règles fondamentales étaient appliquées à la composition et à l'exécution de l'opéra (1981: 335-348). Sans pour autant examiner en détail la question de la pratique instrumentale, il estime que celle-ci n'a pas été écartée : «C'est aux récitatifs surtout que nous percevons la rhétorique de l'opéra. Mais beaucoup de ce que je vais dire s'applique aussi aux airs, et même, si j'ose le dire, à la musique instrumentale » (ibid. : 335).

Mais dans les quelques lignes qu'il consacrera à « la rhétorique dans l'exécution de la musique instrumentale » (ibid. : 347), il relèvera uniquement l'emploi des accents et des rythmes de la déclamation par les instrumentistes, sans considérer la question du corps. Et nul contemporain de Jean Blanchet ne semble avoir proposé de principes philosophiques de l'instrument de musique, comme celui-ci l'avait fait pour le chant. Il fallait dès lors se tourner vers les méthodes destinées aux instruments. Mais que ce soit pour la flûte, le clavecin ou la musette, ou comme ultérieurement pour le violon, la prise en compte du corps dans les méthodes pour instruments de musique des XVIIe et XVIIIe siècle, ne concerne généralement que la manipulation de l'instrument, au sens de prise en main. Il sera cependant parfois question de la tenue de l'instrument comme convenance corporelle. Ainsi, chez François Couperin : «[...] naturellement, pour mouler et former des mains à l'exercice du clavecin, le plutôt est le mieux; et comme la bonne grâce y est nécessaire, il faut commencer par la position du corps. Pour être assis d'une bonne hauteur, il faut que le dessous des coudes, des poignets, et des doigts soit de niveau ; ainsi on doit prendre une chaise qui s'accorde à cette règle. On doit mettre quelque chose de plus, ou de moins haut sous les pieds des jeunes personnes à mesure qu'elles croissent, afin que leurs pieds, n'étant point en l'air, puissent soutenir le corps dans un juste équilibre » (1717: 25-26). Ou bien encore chez Charles-Emmanuel Borjon de Scellery qui, dans sa méthode de musette consacre un chapitre entier aux "grimaces et à la manière de les éviter » (1672:16-18).

31 Grâce, équilibre du corps, juste milieu, tous les principes de la civilité sont reconduits : la question du comportement dans l'espace public n'est pas indifférente aux musiciens. Le corps de l'instrumentiste participe de la musique pour toucher le spectateur, selon Hotteterre le Romain : «Si l'on est debout, il faut être bien campé sur ses jambes, le pied gauche avancé, le corps posé sur la hanche droite ; le tout sans aucune contrainte. On doit surtout observer de ne faire aucun mouvement du corps ni de la tête, comme plusieurs 
font en battant la mesure. Cette attitude étant bien prise, est fort gracieuse et ne prévient pas moins les yeux, que le son de l'instrument flatte agréablement l'oreille» (1722: 5-6).

Le flûtiste se soucie bien de l'instance du témoin, mais n'en développe pas pour autant une actio instrumentale. Faut-il en conclure à l'inexistence de sources écrites en ce domaine, ou bien à la résistance des instrumentistes à la rhétorique ? Je pencherai pour ma part en direction de la première proposition : d'abord parce que la domination de la musique vocale sur la musique instrumentale explique l'absence de documentation sur la question. De plus, si la seconde est bien sous la coupe de la première, on peut y voir une bonne raison pour qu'elle suive les mêmes préceptes. Ensuite parce qu'on peut glaner ici et là quelques indices en faveur de cette hypothèse. Indices picturaux, comme par exemple celui que donne A. P. de Mirimonde, concernant la pratique du luth au XVIIe siècle. Après avoir rappelé que le luth avait acquis un registre " pathétique et sombre ", et qu'il devenait capable de traduire "une rêverie, une mélancolie sincère ou feinte", notamment avec John Dowland, il montre que l'iconographie de l'époque en fournit une image fidèle. C'est ainsi le cas d'un tableau représentant le luthiste Michel de Lasne, celuici : «le visage douloureux égrène sur son luth des arpèges nostalgiques : Afin d'alentir la rigueur/ De celle qui ravit mon cœur/ Soudain dessus mon luth je chante / Les passions que son amour / Me faict ressentir nuit et jour » $(1977: 140)$.

Ici le visage participe de la musique, se mettant en accord avec le contenu des paroles proférées. Dès lors que le chanteur s'accompagne à l'instrument, il n'en abandonne pas pour autant les techniques de l'action chantante. Et s'il joue seulement il fera de même. C'est du moins ce que l'on peut légitimement penser, et ce que confirment des indices littéraires. Ainsi Le neveu de Rameau, imitant un claveciniste, chez Denis Diderot: " Les passions se succédaient sur son visage. On y distinguait la tendresse, la colère, le plaisir, la douleur. On sentait les piano, les forte » $(1984: 37)$. On pourra objecter à bon droit que le "Neveu », en exécutant une pantomime, force le trait, et que seule la dynamique de son visage participe de la réalisation musicale. Mais lorsque Michel-Paul Guy de Chabanon voudra, quelques années plus tard, donner au mélomane un moyen de s'assurer de la qualité d'une mélodie, il convoquera le corps de l'instrumentiste en son entier : « Voulezvous vous assurer d'une manière infaillible si la mélodie de tel musicien a du charme et du caractère? Regardez, écoutez l'orchestre qui l'exécute. S'il s'anime en exécutant, si leurs sons ne sortent point à froid de leur instrument, la mélodie a parlé à leur âme ; cette preuve est sans réplique » (Chabanon $1785: 181$ ).

S'il n'y a pas explicitement d'action musicale instrumentale, dans le sens où Blanchet parlait d'action chantante, il est cependant certain que l'on peut parler de rhétorique du corps du musicien, qui doit beaucoup aux "philosophies du corps" précédemment examinées. Au siècle suivant, au XIXe siècle donc, la lecture de la méthode de violon de Pierre Baillot, fondement de la pédagogie pour cet instrument, conforte cette thèse. L'auteur élabore sa pédagogie en empruntant les notions clefs de la rhétorique, en les adaptant à la pratique musicale instrumentale, entre autres celle de "naturel artistique » issue directement du «naturel étudié » de la rhétorique. Si dès lors, on peut parler d'une rhétorique du corps du musicien, celle-ci peut changer quant à son contenu, selon les époques et en fonction de l'évolution des discours sur le corps, voire aussi des individus. Mais cela est un autre problème. Quoi qu'il en soit, la culture musicale du corps qui se met en place sous l'Ancien Régime pourra ensuite s'épanouir comme comportement « naturel » et, oubliant son origine, s'explicitera comme une emprise de la musique sur le corps du musicien. 


\section{BIBLIOGRAPHIE}

BACILLY Bénigne de, 1971 [1679], L'art de bien chanter. Paris : Lottin. Réédition Minkoff Reprints. BAILLOT Pierre, 1834, L'art du violon. Nouvelle méthode dédiée à ses élèves par P. Baillot, membre de la légion d'honneur, de la musique particulière du Roi et professeur au conservatoire de musique. Paris : Dépôt Central de la musique.

BARNETT David, 1981, « La rhétorique de l'opéra ». XVIIe siècle : 335-348.

BARY René, 1659, La rhétorique française où l'on trouve de nouveaux exemples sur les passions et les figures. Paris : chez Pierre Le Petit.

BEAUVAIS J., 1784, L'art de bien parler et de bien écrire en français. Paris : chez la veuve Valade.

BLANCHET Jean, 1766, L'art ou les principes philosophiques du chant. Deuxième édition. Paris : chez Lottin, Lambert, Duchesne.

BORJON de SCELLERY, 1672, Traité de la musette. Lyon : chez Jean Girin et Bathélémy Rivière.

CALLIERES François de, 1717, De la science du monde et des connaissances utiles à la conduite de la vie. Paris : chez Etienne Ganneau.

CANNONE Belinda, 1990, Philosophies de la musique (1752-1780). Paris : Aux Amateurs de Livres.

CHABANON Michel Paul Guy de, 1785, De la musique considérée en elle-même et dans ses rapports avec la parole, les langues, la poésie et le théâtre. Paris : Pissot.

CICERON, 1974, De officiis (Les devoirs). Paris : Les Belles Lettres.

CONRART Valentin, 1657, Traité de l'action de l'orateur, ou de la prononciation et du geste. Paris : chez Augustin Courbe.

COUPERIN François, 1933 [1717], CEuvres complètes I : Cuvres didactiques. Paris : Editions de l'Oiseau lyre.

COURTIN Antoine de, 1699, Nouveau traité de la civilité qui se pratique en France parmi les honnêtes gens, augmentée de la civilité chrétienne. Toulouse : chez Etienne Manarit.

DIDEROT Denis, 1984, Le neveu de Rameau. Paris : Le Livre de Poche.

DIDIER Béatrice, 1985, La musique des Lumières. Diderot - L'Encyclopédie - Rousseau. Paris : P.U.F.

ELIAS Norbert, 1973, La civilisation des mœurs. Paris: Calmann-Lévy (coll. Agora Presses Pocket).

FRANCE Peter, Mc GOWAN Margaret, 1981, « Autour du « Traité du récitatif » de Grimarest ». XVIIe siècle, 132 : 303-307.

FUMAROLI Marc, 1994 [1980], L'âge de l'éloquence. Paris : Albin Michel.

GOFFMAN Erwin, 1974, Les rites d'interaction. Paris : Editions de Minuit.

GRIMAREST Jean-Léonor Le Gallois de, 1707, Traité du récitatif. Paris : chez Jacques Le Fèvre et Pierre Ribou.

HOTTETERRE LE ROMAIN, 1722, Principes de la flûte traversière ou flûte d'Allemagne, de la flûte à bec ou flûte douce et du hautbois, divisez en différents traités. Paris : J.-B. C. Ballard. 
LAMY le R.P. Bernard, 1715, La rhétorique ou l'art de parler. Nouvelle édition revue et augmentée. Paris : chez Florentin Delaulne.

LE FAUCHEUR, 1667, Traité de l'action de l'orateur ou de la prononciation et du geste. Paris : chez Augustin Courbé.

MABRU Lothaire, 1995, « Postures musiciennes ». Ethnologie Française XXV/4 : 591-607., 1997, « De l'écriture du corps dans les pratiques musicales à transmission « orale » : le cas du fifre en Bazadais ». In N. Belmont et J.-F. Gossiaux : De la voix au texte. L'ethnologie contemporaine entre l'oral et l'écrit. Paris : Editions du Comité des travaux historiques et scientifiques : 49-59., 1999, « Donner à voir la musique : les techniques du corps des violonistes ». In Musurgia, Analyses et pratiques musicales $\mathrm{VI} / 2$ : 29-47.

MAUSS Marcel, 1985, « Les techniques du corps ». In Sociologie et anthropologie. Paris : P.U.F. : 363-386.

MIRIMONDE A. P. de, 1977, L'iconographie musicale sous les rois Bourbons : la musique et les arts plastiques aux XVIIe et XVIIIe siècles. Tome 2. Paris : Picard.

MOLINO Jean, 1988, « Geste et musique : prolégomènes à une anthropologie de la musique ». In Analyse Musicale $10: 8-15$.

ROLLIN Charles, 1845 [1755]De la manière d'enseigner et d'étudier les Belles-Lettres par rapport à l'esprit et au cœur. Tome quatrième, nouvelle édition. Paris : chez les Frères Estienne.

ROUGEMONT Martine de, 1981, « L'acteur et l'orateur : étapes d'un débat ». XVIIe siècle 132 : 329-333.

SALLE Jean-Baptiste de la, 1736, Les règles de la bienséance et de la civilité chrétienne. Paris : chez Gabriel Valleyre.

SCHAEFFNER André, 1936, Origine des instruments de musique. Introduction ethnologique à l'histoire de la musique instrumentale. Paris : Payot.

\section{NOTES}

1. Cf. à ce propos mon article intitulé «Des postures musiciennes" (Mabru 1995: 591-606) qui traite cette question à travers l'étude de violonistes «classiques ", «baroques » et «traditionnels».

2. Le terme de ruelle qui désigne depuis le $\mathrm{XV}^{\mathrm{e}}$ siècle l'espace libre entre le lit et le mur dans une chambre, a pris au XVII ${ }^{\mathrm{e}}$ siècle le sens de salon mondain et littéraire, les femmes de haut rang recevant dans leur chambre à coucher.

3. «Grimarest proclame plus d'une fois la nouveauté de son entreprise. Faut-il l'en croire ? Il est certain que sa théorie se situe sans difficulté dans la grande tradition qui remonte à Cicéron et à Quintilien. Il pille la « Méthode pour bien prononcer un discours et pour le bien animer » (1676) de René Bary, qui s'inspire du traité de l'action (1659) de Le Faucheur- qui lui, proclame sa dette envers Quintilien. Et si l'on compare les idées de Grimarest avec les conseils de Charles Rollin dans son Traité des études (pages toutes inspirées de Quintilien, que Rollin venait d'éditer) on s'aperçoit qu'il y a peu de différences entre les leçons de l'ancien de l'université et celles de notre écrivain très médiocre [selon Rollin] » (France \& Mc Gowan $1981: 304$ ).

4. Si Blanchet élabore et développe la notion d'action chantante, l'idée d'utiliser les préceptes de la rhétorique dans le chant n'est cependant pas neuve. Marin Mersenne recommandait plus d'un siècle auparavant au chanteurs de lire Quintilien: "Vous pouvez en chercher le reste de Quintilien, et d'autres rhétoriciens, de l'enseignement desquels on peut former des gestes 
convenables à la musique même. [...] Ces choses que peut demander Fabius [Quintilien] à un orateur, j'ose les demander à la musique, pour que le chant soit parfaitement émouvant, et arrive à ses fins » (cité in Barnett $1981: 348$ ).

5. Ainsi l'abbé de Condillac, dans son Essai sur l'origine des connaissances humaines dont la première édition date de 1746, écrira à propos des pantomimes chez les Romains : "Elles avaient même l'avantage de plaire beaucoup plus, parce que l'imagination est plus vivement affectée d'un langage qui est tout en action» $(1777: 221)$. Au contraire, la musique de son temps lui semble avoir moins de pouvoir sur les auditeurs : « [...] au moment où elle s'exécute, nous gardons tout le sang froid dont nous sommes capables; nous n'aidons point le musicien à nous en retirer, et les sentiments que nous éprouvons naissent uniquement de l'action des sons sur les oreilles » (ibid .:232).

6. Cf. à ce propos Didier 1985 : 298, et ce que j'en dis concernant le violon dans Mabru 1995, au paragraphe intitulé « Une métaphore de la musique et de la voix ».

\section{RÉSUMÉS}

La question du geste et, plus globalement, du corps dans la musique ne reçoit pas autant d'attention qu'elle le mérite. Et surtout elle est souvent traitée dans une perspective psychologisante, par le recours à la notion d'intériorité. Du point de vue développé ici cette dernière semble inutile. C'est du moins ce que l'on voudra montrer en examinant comment s'est constituée dans notre société une "culture musicale du corps", qui s'inscrit dans une histoire plus générale du corps. Une histoire du corps que l'on examine dans un temps long (XVIe-XVIIIe siècles) et qui permet de montrer que les différentes "théories" du corps élaborées à cette époque (la civilité, la rhétorique, voire la physiognomonie) postulent une relation étroite entre le corps - l'extériorité - et la part spirituelle de l'homme - l'intériorité. Dès lors elles mettront en place dans une profusion de traités des règles comportementales que l'honnête homme devra suivre, et qui passeront ensuite dans la pratique musicale vocale, puis instrumentale.

\section{AUTEUR}

\section{LOTHAIRE MABRU}

Lothaire MABRU, né en 1955, est maître de conférences à l'Université Michel de Montaigne/ Bordeaux 3, où il enseigne l'ethnomusicologie. Il a débuté ses activités professionnelles par une ethnographie des pratiques musicales en milieu rural pour élargir ensuite son domaine d'investigation à une anthropologie de la musique. Sa thèse soutenue en 1995 a l'école des Hautes Etudes en Sciences Sociales de Paris, et intitulée « Du fifre au violon, introduction à une ethnologie du corps dans la musique », pose la question du corps dans la pratique musicale instrumentale ; elle examine le rôle du corps dans l'apprentissage et la transmission du savoir dans une pratique sans écriture, mais aussi dans la mise en visibilité de la musique dans l'espace public. Il a publié plusieurs articles et participé à des ouvrages collectifs sur les problèmes de la transmission du savoir, de l'oralité et de l'écriture, et bien évidemment sur la question du corps. Il est aussi co-éditeur des travaux de Félix Arnaudin dans le domaine du folklore musical, et auteur d'un ouvrage visant une approche anthropologique de la lutherie en France. Musicien, il 
pratique le violon et la mandoline, ainsi que la vielle à roue. Il a joué avec Lubat père et fils, Robert Amyot, et se produit actuellement en duo avec Patrick Cadeillan.fr 